\title{
HYPSARRHYTHMIA VARIANT PATTERNS
}

The frequency of hypsarrhythmia variant or atypical patterns was determined by analysis of pre-ACTH EEG records of 53 consecutive patients with infantile spasms at Children's Hospital, Harvard Medical School, Boston, MA. Hypsarrhythmia was scored (16 most severe, 0 normal EEG) based on degree of disorganization, diffuse delta, voltage, spikes, burst suppression (BS), etc. Variant patterns occurred in $69 \%$ of records. Hemihypsarrhythmia or BS was characteristic of spasms caused by cerebral dysgenesis. Sleep patterns were persistently abnormal in infants with perinatal hypoxic-ischemic encephalopathy. Lower hypsarrhythmia scores $(<10),<75 \%$ delta activity, and absence of electrodecremental discharges correlated with more favorable outcome, whereas variant patterns had no prognostic significance. (Kramer U, Sue WC, Mikati MA. Hypsarrhythmia: Frequency of variant patterns and correlation with etiology and outcome. Neurology Jan 1997;48:197-203). (Reprints: Dr MA Mikati, Department of Pediatrics, c/o American University of Beirut New York Office, 850 Third Ave, New York, NY 10022).

COMMENT. Analysis of pre-ACTH EEGs in patients with West syndrome may reflect the pathophysiology and prognosis. Higher severity scores of hypsarrhythmia correlate with poor outcome. Variant patterns of hypsarrhythmia are frequent and show no correlation with prognosis; they should be included within the definition and indications for treatment with ACTH.

Cortical hypometabolism and delayed myelination were reported in a study of 18 children with West syndrome at Nagoya University School of Medicine, Japan. (Natsume J, Watanabe K, Maeda N et al. Epilepsia Dec $1996 ; 37: 1180-1184)$. Hypometabolism on PET scans at the onset of spasms or at 10 months is correlated with MRI evidence of delayed myelination at age 10 months.

\section{POSITIVE SPIKES WITH NEURONAL MIGRATION DISORDERS}

Five of fifteen children with histologically proven neuronal migration disorders (NMD) and refractory localization-related epilepsy had surface positive spikes or sharp waves or both on scalp EEG performed preoperatively in a study at The Hospital for Sick Children, Toronto, Canada. Patients with positive epileptiform discharges had earlier onset of seizures with focal motor pattern, poor response to AEDs, a higher frequency of hemiparesis preoperatively, and surgery at a younger age with poor outcome. Lesions involving the rolandic fissure are more extended than those seen on the MRI and cannot be completely resected. (Otsubo H, Steinlin M, Hwang PA, et al. Positive epileptiform discharges in children with neuronal migration disorders. Pediatr Neurol Jan 1997;16:23-31). (Respond: Dr Otsubo, EEG Laboratory, Division of Neurology, Hospital fro Sick Children, 555 University Ave, Toronto, Ontario M5G 1X8, Canada).

COMMENT. Compared to the usual negative polarity of most epileptiform abnormalities, focal positive spike waves encountered with neuronal migration disorders are predictive of more extensive lesions, higher incidence of hemiparesis, focal motor seizures refractory to AEDs, and less favorable surgical outcome associated with incomplete resection.

The pathomechanism of the neuronal migration disorder seen in Miller-Dieker syndrome (smooth cerebral surface and four 
layered thickened cortex) was investigated using specific antibodies against the protein product of LIS-1, the gene responsible for MDS phenotype, at the National Center of Neurology and Psychiatry, Kodaira, Tokyo. (Isumi H, Takashima S, Kakita A, et al. Pediatr Neurol Jan 1997;16:42-44). Loss of LIS-1 immunoreactivity occurred in brains with MDS, but not with isolated lissencephaly, holoprosencephaly, Fukuyama-type congenital muscular dystrophy, and Zellweger syndrome. Loss of LIS-1 gene product is specific to the abnormal neuronal migration in Miller-Dieker syndrome.

\section{UNTREATED TONIC-CLONIC SEIZURE OUTCOME}

The clinical course of untreated tonic-clonic seizures in 204 children aged 1 month to 16 years was studied at the University Hospital Rotterdam, Netherlands. Follow-up continued until start of drug treatment (78), the fourth untreated seizure (41), or for two years without treatment (85). Forty-two per cent had a decelerating pattern; they were free of seizures despite treatment being withheld. Of 41 with four or more untreated seizures, 8 had an accelerating pattern, the intervals between seizures becoming shorter. In 110 children the disease process could not be classified because treatment was started after the first, second, or third seizure. (Van Donselaar CA, Brouwer OF, Geerts AT, et al. Clinical course of untreated tonic-clonic seizures in childhood: prospective, hospital based study. BMI 8 Feb 1997;314:401-404). (Respond: Dr CA van Donselaar, St Clara Hospital, Olympiaweg 350, 3078 HT Rotterdam, Netherlands).

COMMENT. Previous reports of an accelerating pattern, or decreasing interval between successive untreated seizures, support the concept that seizures beget seizures (Gowers, 1881) and early introduction of antiepileptic drug treatment is recommended. The present study fails to confirm the fear that untreated tonic-clonic seizures will evolve into a progressive disorder, and favors a delay in treatment. An individualized approach is probably most appropriate, each child's treatment based on factors predictive of seizure recurrence. For further reference to treatment onset and epilepsy prognosis, see Progress in Pediatric Neurology II, PNB Publ, 1994:pp92-93.

The risk for psychiatric and psychosomatic disorders is higher than expected in adults with childhood-onset epilepsy, regardless of continued treatment with AEDs, in a study at the University of Turku, Finland. (Jalava M, Sillanpaa M. Concurrent illnesses in adults with childhood-onset epilepsy: a population-based 35-year follow-up study. Epilepsia Dec 1996;37:1155-1163).

\section{BENIGN EPILEPSY WITH C-T SPIKES (BECT) OUTCOME}

A meta-analysis of 13 cohorts, comprising 794 patients, selected according to ILAE and other criteria from 525 publications on BECT, was conducted at Leiden University Hospital, Netherlands. Age at onset ranged from 3 months to 14 years, and age at last seizure ranged from 3 to 18 years. At an older age, the proportion of patients in remission was 0.9997. Despite this apparent excellent prognosis, the outcome of BECT in a child just developing seizures could not be determined satisfactorily from the meta-analysis, because of the retrospective design and unclear selection of patients studied. (Bouma PAD, Bovenkerk AC, Westendorp RGJ, Brouwer OF. The course of benign partial epilepsy of childhood with centrotemporal spikes: a meta-analysis. Neurology Feb 1997;48:430-437). (Reprints: Dr PAD Bouma, Department of Neurology, Leiden University Hospital, PO Box 9600, 2300 RC Leiden, Netherlands).

COMMENT. AED treatment was started in $81.6 \%$ of the patients in the 